\title{
Neutralizing and non-neutralizing antibody responses in HIV-1 subtype C chronically infected patients with divergent rates of disease progression
}

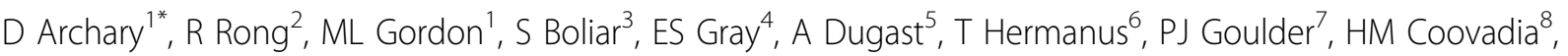

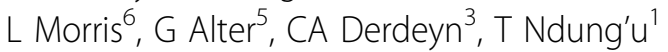

From AIDS Vaccine 2012

Boston, MA, USA. 9-12 September 2012

\section{Background}

Development of an efficacious HIV-1 vaccine able to elicit the production of broadly neutralizing antibodies (nAbs), capable of retaining potent activity against a diverse panel of viral isolates remains a significant challenge. The evolutionary forces that shape envelope and ensuing $\mathrm{nAb}$ and non-neutralizing antibodies in HIV-1 subtype $\mathrm{C}$ are incompletely understood and these two parameters have been rarely studied concurrently.

\section{Methods}

We characterized patterns of virus-specific nAbs and nonneutralizing antibodies in four slow progressors and four progressors with chronic HIV-1 subtype C infection, over a median of 21 months. Single cycle neutralization assays was performed. In addition, the binding affinities of HIVspecific immunoglobulins (IgGs) and the affinities of the IgGs to various $\mathrm{Fc} \gamma$ receptors (Fc $\gamma \mathrm{Rs}$ ) were assessed.

\section{Results}

NAbs evolved significantly in progressors $(\mathrm{p}=0.003)$ from study entry to study exit. NAb IC50 titers significantly correlated with amino acid lengths for V1-V2 ( $\mathrm{p}=0.04)$, C3V5 $(\mathrm{p}=0.03)$ and V1-V5 $(\mathrm{p}=0.04)$. Both groups displayed preferential heterologous activity against the subtype $\mathrm{C}$ panel. Both groups displayed preferential heterologous activity against the subtype $C$ panel. There were no significant differences in breadth of responses between the groups for either subtype A or C. Neutralization breadth

${ }^{1}$ University of KwaZulu-Natal, Durban, South Africa

Full list of author information is available at the end of the article and titers to subtype B reference strains was significantly higher in progressors compared to slow progressors (both $\mathrm{p}<0.03$ ) with increasing $\mathrm{nAb}$ breadth from study entry to study exit in progressors. Progressors had cross-reactive neutralizing antibodies that targeted V2 and V3. Binding affinities of non-neutralizing antibodies to HIV-specific gp120, gp41 and p24 and to activating and inhibitory Fc $\gamma$ receptors (Fc $\gamma$ Rs) were similar in both groups. However, in slow progressors, CD4 T-cell counts correlated inversely with antibody binding affinity for the activating Fc $\gamma$ RIIa $(\mathrm{p}=0.005)$.

\section{Conclusion}

Overall, the data suggest that neither nAbs nor nonneutralizing antibodies could be directly associated with disease attenuation. However, continuous evolution of nAbs was a potential marker of disease progression.

\section{Author details}

'University of KwaZulu-Natal, Durban, South Africa. ${ }^{2}$ Jiaotong-Liverpool University, Suzhou, China, China. ${ }^{3}$ Emory University, Atlanta, GA, USA. ${ }^{4}$ University of Western Australia, Australia. ${ }^{5}$ Ragon Institute, Boston, MA, USA. ${ }^{6}$ National Institute for Communicable Diseases, Johannesburg, South Africa. ${ }^{7}$ Oxford University, UK. ${ }^{8}$ University of KwaZulu Natal, Durban, South Africa.

Published: 13 September 2012

doi:10.1186/1742-4690-9-S2-P75

Cite this article as: Archary et al:: Neutralizing and non-neutralizing antibody responses in HIV-1 subtype C chronically infected patients with divergent rates of disease progression. Retrovirology 2012 9(Suppl 2):P75. 